\title{
A method to decipher pleiotropy by detecting underlying heterogeneity driven by hidden subgroups applied to autoimmune and neuropsychiatric diseases DOI:
}

10.1038/ng.3572

\section{Document Version \\ Accepted author manuscript}

Link to publication record in Manchester Research Explorer

Citation for published version (APA):

Han, B., Pouget, J. G., Slowikowski, K., Stahl, E., Lee, C. H., Diogo, D., Hu, X., Park, Y. R., Kim, E., Gregersen, P. K., Dahlqvist, S. R., Worthington, J., Martin, J., Eyre, S., Klareskog, L., Huizinga, T., Chen, W-M., OnengutGumuscu, S., Rich, S. S., ... Raychaudhuri, S. (2016). A method to decipher pleiotropy by detecting underlying heterogeneity driven by hidden subgroups applied to autoimmune and neuropsychiatric diseases. Nature Genetics, 48(7), 803 - 810. https://doi.org/10.1038/ng.3572

Published in:

Nature Genetics

\section{Citing this paper}

Please note that where the full-text provided on Manchester Research Explorer is the Author Accepted Manuscript or Proof version this may differ from the final Published version. If citing, it is advised that you check and use the publisher's definitive version.

\section{General rights}

Copyright and moral rights for the publications made accessible in the Research Explorer are retained by the authors and/or other copyright owners and it is a condition of accessing publications that users recognise and abide by the legal requirements associated with these rights.

\section{Takedown policy}

If you believe that this document breaches copyright please refer to the University of Manchester's Takedown Procedures [http://man.ac.uk/04Y6Bo] or contact uml.scholarlycommunications@manchester.ac.uk providing relevant details, so we can investigate your claim.

\section{OPEN ACCESS}




\title{
A method to decipher pleiotropy by detecting underlying heterogeneity driven by hidden subgroups applied to autoimmune and neuropsychiatric diseases
}

\author{
Buhm Han ${ }^{1-4,25}$, Jennie G Pouget ${ }^{1,5-7,25}$, Kamil Slowikowski ${ }^{1,3,4,8}$, Eli Stahl' ${ }^{9}$, Cue Hyunkyu Lee ${ }^{10}$, Dorothee Diogo ${ }^{1,3,4}$, \\ Xinli Hu ${ }^{1,3,4,11}$, Yu Rang Park ${ }^{10,12}$, Eunji Kim ${ }^{10,13}$, Peter K Gregersen ${ }^{14}$, Solbritt Rantapää Dahlqvist ${ }^{15}$, \\ Jane Worthington ${ }^{16,17}$, Javier Martin ${ }^{18}$, Steve Eyre ${ }^{16,17}$, Lars Klareskog ${ }^{19}$, Tom Huizinga ${ }^{20}$, Wei-Min Chen ${ }^{21}$, \\ Suna Onengut-Gumuscu ${ }^{21}$, Stephen S Rich ${ }^{21}$, Major Depressive Disorder Working Group of the Psychiatric \\ Genomics Consortium $^{22}$, Naomi R Wray ${ }^{23}$ \& Soumya Raychaudhuri ${ }^{1,3,4,19,24}$
}

There is growing evidence of shared risk alleles for complex traits (pleiotropy), including autoimmune and neuropsychiatric diseases. This might be due to sharing among all individuals (whole-group pleiotropy) or a subset of individuals in a genetically heterogeneous cohort (subgroup heterogeneity). Here we describe the use of a well-powered statistic, BUHMBOX, to distinguish between those two situations using genotype data. We observed a shared genetic basis for 11 autoimmune diseases and type 1 diabetes (T1D; $P<1 \times 10^{-4}$ ) and for 11 autoimmune diseases and rheumatoid arthritis $\left(\mathrm{RA} ; P<1 \times \mathbf{1 0}^{-3}\right)$. This sharing was not explained by subgroup heterogeneity (corrected $\boldsymbol{P}_{\text {BUнмвох }}$ $>0.2 ; 6,670 \mathrm{~T} 1 \mathrm{D}$ cases and 7,279 RA cases). Genetic sharing between seronegative and seropostive RA $\left(P<1 \times 1^{-9}\right)$ had significant evidence of subgroup heterogeneity, suggesting a subgroup of seropositive-like cases within seronegative cases $\left(\boldsymbol{P}_{\text {Buнmвох }}=\mathbf{0 . 0 0 8} ; \mathbf{2 , 4 0 6}\right.$ seronegative $\mathrm{RA}$ cases $)$. We also observed a shared genetic basis for major depressive disorder (MDD) and schizophrenia $\left(P<1 \times 1^{-4}\right)$ that was not explained by subgroup heterogeneity $\left(P_{\mathrm{BUHMBOX}}=\mathbf{0 . 2 8}\right.$; 9,238 MDD cases).
Recent studies have demonstrated that many diseases have risk alleles in common ${ }^{1-4}$ and exhibit significant coheritability ${ }^{5-7}$. Coheritability studies are defining the relationship between complex traits and providing new insights into disease mechanisms. As more phenotypes are studied using genetics in the context of emerging deeply phenotyped population-wide cohorts ${ }^{8}$, including the Precision Medicine Initiative ${ }^{9}$, coheritability of traits will become even more apparent. In the genomics era, methods for detecting coheritability have moved beyond traditional approaches such as twin or family studies ${ }^{10,11}$. Now, alternative approaches using genome-wide association study (GWAS) data from unrelated individuals are widely used. Polygenic risk score approaches ${ }^{3,12,13}$ build genetic risk scores (GRSs) for one phenotype and test the association of those GRSs with a second phenotype. Mixed-model approaches $5,6,14$ can be used to estimate the genetic covariance of two traits on the observed scale. Genetic covariance can be used to calculate genetic correlation and coheritability ${ }^{6}$. Cross-trait linkage disequilibrium score regression (LDSC) uses linkage disequilibrium (LD) and summary statistics obtained from GWAS to estimate the genetic correlation attributable to SNPs ${ }^{7}$. In addition, the $P$ values of independent SNPs associated with multiple phenotypes can be tested for a significant deviation from the null distribution ${ }^{2}$.

\footnotetext{
${ }^{1}$ Department of Medicine, Brigham and Women's Hospital and Harvard Medical School, Boston, Massachusetts, USA. ${ }^{2}$ Department of Convergence Medicine, University of Ulsan College of Medicine and Asan Institute for Life Sciences, Asan Medical Center, Seoul, Republic of Korea. ${ }^{3}$ Program in Medical and Population Genetics, Broad Institute of MIT and Harvard, Cambridge, Massachusetts, USA. ${ }^{4}$ Partners Center for Personalized Genetic Medicine, Boston, Massachusetts, USA. ${ }^{5}$ Campbell Family Mental Health Research Institute, Centre for Addiction and Mental Health, Toronto, Ontario, Canada. ${ }^{6}$ Institute of Medical Science, University of Toronto, Toronto, Ontario, Canada. ${ }^{7}$ Department of Psychiatry, University of Toronto, Toronto, Ontario, Canada. ${ }^{8}$ Bioinformatics and Integrative Genomics, Harvard University, Cambridge, Massachusetts, USA. ${ }^{9}$ Department of Psychiatry, Mount Sinai School of Medicine, New York, New York, USA. ${ }^{0}$ Asan Institute for Life Sciences, University of Ulsan College of Medicine, Asan Medical Center, Seoul, Republic of Korea. ${ }^{11}$ Harvard-MIT Division of Health Sciences and Technology, Boston, Massachusetts, USA. ${ }^{12}$ Department of Biomedical Informatics, Asan Medical Center, Seoul, Republic of Korea. ${ }^{13}$ Department of Chemistry, Seoul National University, Seoul, Republic of Korea. ${ }^{14}$ Robert S. Boas Center for Genomics and Human Genetics, Feinstein Institute for Medical Research, Manhasset, New York, USA. ${ }^{15}$ Department of Public Health and Clinical Medicine, Rheumatology, Umeå University, Umeå, Sweden. ${ }^{16}$ Arthritis Research UK Centre for Genetics and Genomics, Musculoskeletal Research Centre, Institute for Inflammation and Repair, Manchester Academic Health Science Centre, University of Manchester, Manchester, UK. ${ }^{17}$ National Institute for Health Research, Manchester Musculoskeletal Biomedical Research Unit, Central Manchester University Hospitals National Health Service Foundation Trust, Manchester Academic Health Sciences Centre, Manchester, UK. ${ }^{18}$ Instituto de Parasitología y Biomedicina López-Neyra, Consejo Superior de Investigaciones Científicas, Granada, Spain. ${ }^{19}$ Rheumatology Unit, Department of Medicine, Karolinska Institutet and Karolinska University Hospital Solna, Stockholm, Sweden. ${ }^{20}$ Department of Rheumatology, Leiden University Medical Centre, Leiden, the Netherlands. ${ }^{21}$ Center for Public Health Genomics, University of Virginia, Charlottesville, Virginia, USA. ${ }^{22}$ A full list of members and affiliations appears in the Supplementary Note. ${ }^{23}$ Queensland Brain Institute, University of Queensland, Brisbane, Queensland, Australia. ${ }^{24}$ Institute of Inflammation and Repair, University of Manchester, Manchester, UK. ${ }^{25}$ These authors contributed equally to this work. Correspondence should be addressed to S.R. (soumya@broadinstitute.org) or B.H. (buhm.han@amc.seoul.kr).
} 
These approaches have been applied to demonstrate significant shared genetic structure among many phenotypes $5,7,15$, including autoimmune $^{2}$ and neuropsychiatric diseases ${ }^{3,6,13}$. The observed coheritability and genetic sharing suggest the possibility of pleiotropy, defined here as the sharing of risk alleles across traits at specific loci or at a genome-wide level. An example of a variant showing pleiotropy is the PTPN22 variant encoding p.Arg620Trp, which is associated with multiple autoimmune diseases ${ }^{16}$.

The sharing of risk alleles across diseases can be driven by all individuals or by a subset of individuals. In the former scenario, the sharing is clearly driven by pleiotropy (whole-group pleiotropy). In the latter scenario, only a subset of individuals with one disease is genetically similar to individuals with another disease. We call this subgroup heterogeneity, a situation where a patient cohort consists of genetically distinct subgroups that may or may not have distinct symptom profiles and treatment outcomes. Subgroup heterogeneity can occur in the context of misclassifications (for example, when cases with atypical clinical presentations for a different disease are erroneously included), molecular subtypes (for example, when two different etiologies underlie a disease, resulting in a subset of cases that share pathogenesis with a different disease), asymmetric causal relationships (for example, when one disease causes another disease, resulting in a subset of cases that also have the causal disease; often called mediated pleiotropy), or ascertainment bias (for example, when cases also affected with a different disease are more likely to come to clinical attention and be included in the study). Such situations result in a subset of cases that is genetically similar to individuals with another disease, creating shared genetic structure ${ }^{17}$. Indeed, there is now evidence that misclassifications ${ }^{18-21}$, etiological diversity ${ }^{22}$, and ascertainment bias ${ }^{23}$ are prevalent across certain human diseases, leading to the conclusion that significant heterogeneity may exist ${ }^{24-27}$. Because the potential contribution of subgroup heterogeneity to any genetic sharing observed between diseases represents a critical disease insight, statistical methods are needed a
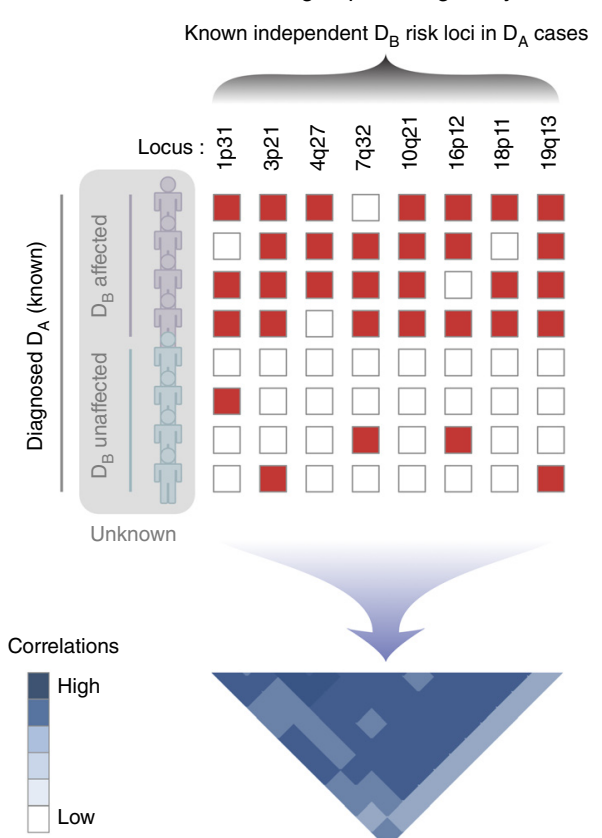

b

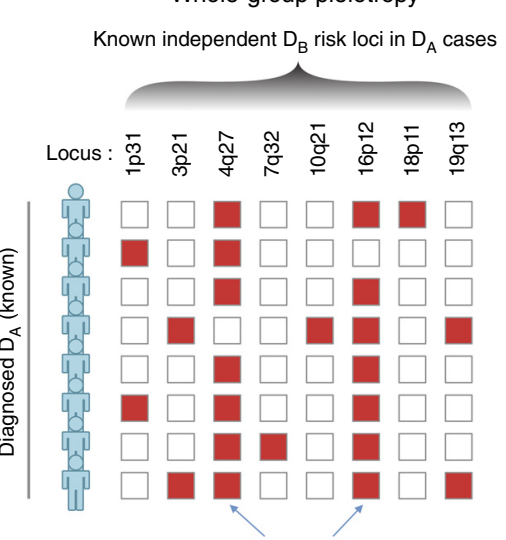

Pleiotropic loci

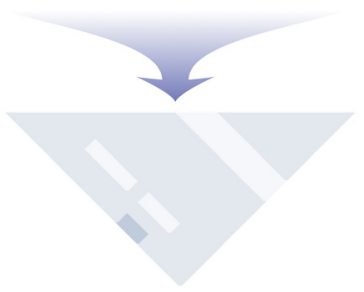

to distinguish subgroup heterogeneity from whole-group pleiotropy. For the purposes of this paper, we will use the term 'pleiotropy' to refer to whole-group pleiotropy and 'heterogeneity' to refer to subgroup heterogeneity.

\section{RESULTS}

\section{Overview of BUHMBOX}

Genetic sharing between disease $A\left(D_{A}\right)$ and disease $B\left(D_{B}\right)$ could be due to pleiotropy but could also be due to heterogeneity (that is, where a subset of $D_{A}$ cases are genetically more similar to $D_{B}$ cases). If we calculated GRSs for $D_{A}$ cases using $D_{B}$-associated loci and their effect sizes $\left(\mathrm{GRS}_{\mathrm{B}}\right)$, the mean of $\mathrm{GRS}_{\mathrm{B}}$ would be statistically different between $D_{A}$ cases and controls under either pleiotropy or heterogeneity. Under pleiotropy, some $\mathrm{D}_{B}$ risk alleles impose risk of $\mathrm{D}_{\mathrm{A}}$, and $\mathrm{D}_{\mathrm{B}}$ risk alleles will be enriched in $D_{A}$ cases compared to controls. Under heterogeneity, a subset of $\mathrm{D}_{\mathrm{A}}$ cases will have genetic characteristics of $D_{B}$, and therefore $D_{B}$ risk alleles will also be enriched in these individuals. In both situations, the enrichment of $D_{B}$ risk alleles in $\mathrm{D}_{\mathrm{A}}$ cases will result in an increased mean $\mathrm{GRS}_{\mathrm{B}}$ in individuals that are $\mathrm{D}_{\mathrm{A}}$ cases. For the same reasons, if we calculated the genetic correlation $\left(r_{\mathrm{g}}\right)$ of $\mathrm{D}_{\mathrm{A}}$ and $\mathrm{D}_{\mathrm{B}}$ using cross-trait $\mathrm{LDSC}^{7}$ in this scenario, the $r_{\mathrm{g}}$ would be positive under both pleiotropy and heterogeneity.

To detect heterogeneity, even in the presence of pleiotropy, we developed BUHMBOX (Breaking Up Heterogeneous Mixture Based On $\operatorname{cross}(\mathrm{X})$-locus correlations). Our method tests for the presence of heterogeneous subgroups (that is, $\mathrm{D}_{\mathrm{B}}$-like cases) in an otherwise homogeneous phenotype (that is, $\mathrm{D}_{\mathrm{A}}$ ). To do this, BUHMBOX requires (i) a list of known $D_{B}$-associated SNPs with corresponding risk alleles, risk allele frequencies (RAFs), and effect sizes and (ii) individual-level genotype data for $D_{B}$ SNPs in $D_{A}$ cases. BUHMBOX leverages the fact that, in the setting of heterogeneity, $D_{B}$ risk alleles have higher allele frequencies only in a specific subset of $\mathrm{D}_{\mathrm{A}}$ cases. In contrast, under true pleiotropy, $\mathrm{D}_{\mathrm{B}}$ risk alleles are expected to have higher allele frequencies across all $D_{A}$ cases (Fig. 1). If $D_{B}$ risk alleles are enriched in one subgroup, the expected correlations of risk allele dosages between loci will be consistently positive (Supplementary Table 1 and Supplementary Note). BUHMBOX combines these pairwise correlations into one statistic and tests for excessive positive correlations. Heterogeneity can lead to a significant BUHMBOX test statistic. In contrast, a lack of true heterogeneity or insufficient power to detect the presence of heterogeneity (type II error) can lead to a nonsignificant BUHMBOX test statistic. Insufficient power occurs when the number of $\mathrm{D}_{\mathrm{A}}$ cases, heterogeneity proportion, or number of known risk alleles and/or their effect sizes for $D_{B}$ are small.

Figure 1 Overview of BUHMBOX. (a) Under the scenario of subgroup heterogeneity, risk alleles for $D_{B}$-associated loci will be enriched in a subgroup of $D_{A}$ cases, producing positive correlations between $D_{B}$ risk allele dosages from independent loci. (b) Under the scenario where there is no heterogeneity and where $D_{A}$ and $D_{B}$ share alleles owing to pleiotropy (whole-group pleiotropy), $D_{B}$ risk alleles will be uniformly distributed and have no

No correlations correlations. Red boxes, $D_{B}$ risk alleles; white boxes, non-risk alleles. 
Figure 2 Power gain by weighting SNPs by allele frequency and effect size. Comparison of the statistical power of BUHMBOX with a weighting scheme that optimally weights correlations between SNPs (weighted) to that in an alternative approach that weights correlations uniformly (unweighted; equation (12) in the Supplementary Note). We simulated 1,000 case individuals and assumed 50 risk loci, whose ORs and RAFs were sampled from the GWAS catalog. Colored bands denote the 95\% confidence intervals of the power estimates.

BUHMBOX discriminates between heterogeneity and pleiotropy To demonstrate that BUHMBOX detects heterogeneity (even in the presence of pleiotropy), we conducted simulations with the following parameters: the sample size of $\mathrm{D}_{\mathrm{A}}$ case individuals $(N)$, the number of risk loci associated with $\mathrm{D}_{\mathrm{B}}(M)$, and the proportion of $\mathrm{D}_{\mathrm{A}}$ cases that actually show genetic characteristics of $D_{B}$ (heterogeneity proportion, or $\pi$ ). To simulate realistic distributions of effect sizes and allele frequencies, we sampled odds ratio (OR) and RAF pairs from reported associations in the National Human Genome Research Insitute (NHGRI) catalog of published GWAS 28 (Online Methods).

To characterize the false positive rate (FPR) of BUHMBOX, we simulated $1,000,000$ studies $(N=2,000$ and $M=50)$ in which there was no heterogeneity ( $\pi=0$; Online Methods) or pleiotropy. BUHMBOX obtained a $5.1 \%$ FPR at $P<0.05$; it also obtained appropriate FPRs at a wide range of statistical significance thresholds $(P<0.05$ to $P<0.0005$; Supplementary Table 2).

To evaluate the FPR of BUHMBOX when there was pleiotropy without heterogeneity $(\pi=0)$, we simulated 1,000 studies $(N=2,000$ and $M=50$ ) assuming that $\mathrm{D}_{\mathrm{A}}$ and $\mathrm{D}_{\mathrm{B}}$ shared $10 \%$ of risk loci (5 loci). We quantified the proportion of instances in which BUHMBOX and GRS approaches obtained $P$ values smaller than the threshold $P<0.05$. The GRS approach appropriately demonstrated $64.8 \%$ power to detect shared genetic structure. BUHMBOX demonstrated an appropriate FPR of $4.3 \%$ in detecting heterogeneity (Supplementary Fig. 1).

Finally, to evaluate the power of BUHMBOX to detect heterogeneity, we repeated these simulations assuming no pleiotropy but subtle heterogeneity. We assumed that $10 \%$ of $\mathrm{D}_{\mathrm{A}}$ cases were actually $\mathrm{D}_{\mathrm{B}}$ cases $(\pi=0.1)$. In this case, BUHMBOX demonstrated $81.7 \%$ power to detect heterogeneity at $P<0.05$ (Supplementary Fig. 1), and the GRS approach demonstrated $100 \%$ power to detect shared genetic structure. Note that the difference in power for the GRS approach in the pleiotropy and heterogeneity simulations is due to the stochastic chance that sampled effect sizes for all five loci may be small in the pleiotropy simulation; in simulations where we fixed the OR (1.25) and RAF (0.3) for all loci, the power of the GRS approach was similar for the two scenarios: $91.8 \%$ in pleiotropy and $92.0 \%$ in heterogeneity.

These simulations illustrate that BUHMBOX is sensitive to heterogeneity but robust to pleiotropy, whereas the GRS approach detects both scenarios and cannot be used to discriminate between the two. Thus,

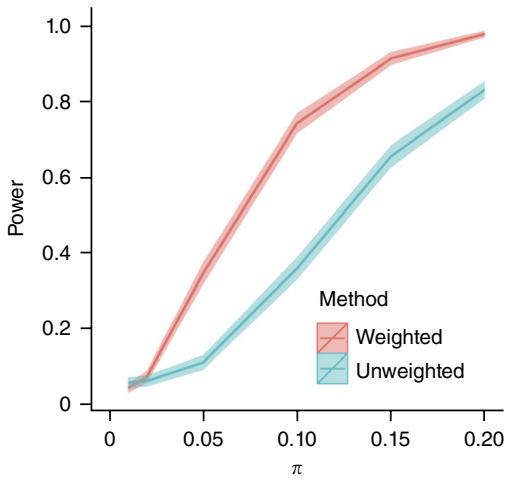

BUHMBOX complements methods for detecting pleiotropy by helping to interpret shared genetic structure (Supplementary Table 1).

\section{Weighting pairwise correlations increases power}

BUHMBOX combines multiple pairwise correlations into one statistic. A pair of loci with larger allele frequencies and effect sizes will show larger expected correlation given the same $\pi$ and may be more informative than other pairs of loci (Supplementary Fig. 2). We hypothesized that accounting for this unequal information between SNP pairs could increase power. We defined a scheme to weight pairwise correlations between loci as a function of their effect sizes and allele frequencies (Online Methods). In simulations, we observed substantial power gain with this weighting scheme. Assuming 1,000 cases and 50 risk loci, we compared power for BUHMBOX implemented with and without weighting correlations (equation (12) in the Supplementary Note). Across a wide range of $\pi$ values, we observed that weighting dramatically increased power (Fig. 2). For example, at $\pi=0.1$, the weighted implementation of BUHMBOX yielded $74 \%$ power, in comparison to the unweighted implementation, which yielded only $36 \%$ power.

\section{Power is proportional to number of samples and loci}

The statistical power of BUHMBOX is a function of many factors, including sample size $N$ of the cases we are testing for heterogeneity, $\pi$, $M$, RAF, and OR. We sampled pairs of RAF and OR values from the GWAS catalog. Given a sample size of $N=2,000$ cases and 2,000 controls, assuming $\pi=0.2$ and 50 risk loci, BUHMBOX achieved $92 \%$ power at $P<0.05$ (Fig. 3). As many GWAS now consist of more than 2,000 cases and many diseases are approaching 50 known associated $\operatorname{loci}^{28}$, BUHMBOX is currently well powered to detect a moderate amount of heterogeneity $(\pi=0.2)$ for many human traits. Modest heterogeneity is more challenging to detect at this sample size; power decreased to $67 \%$ at $\pi=0.1$ and to $38 \%$ at $\pi=0.05$. Power can be augmented with larger sample size (Fig. 3) and larger effect sizes (Supplementary Fig. 3). Power can also be increased by including
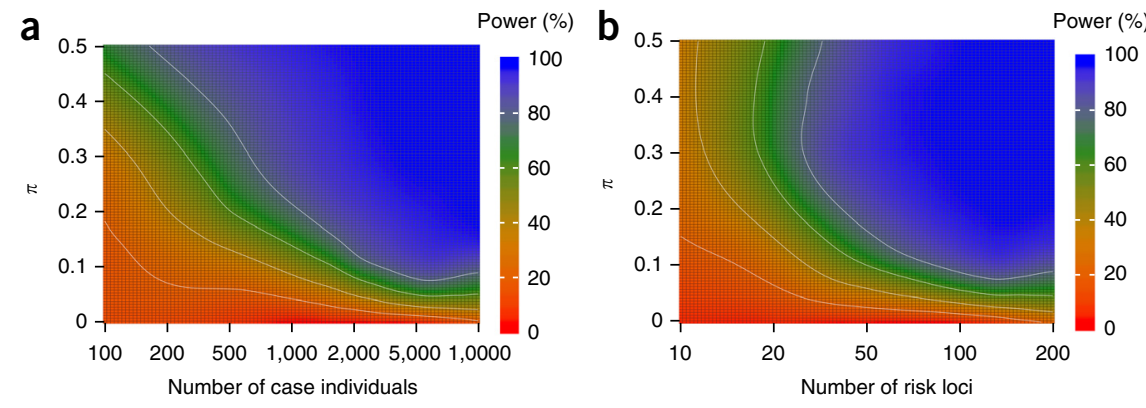

Figure 3 Power of BUHMBOX for detecting heterogeneity as a function of the number of risk loci, the number of case samples, and the proportion of samples that actually have a different phenotype $(\pi)$. $(\mathbf{a}, \mathbf{b})$ Power as a function of the number of case individuals and heterogeneity proportion, when the number of risk loci was fixed at 50 (a), and of the number of risk loci and heterogeneity proportion, when the case sample size was fixed at 2,000 (b). We assumed that we had the same number of controls as cases. White lines denote $20 \%, 40 \%, 60 \%$, and $80 \%$ power. 
TECHNICAL REPORTS

Table 1 Summary of genetic overlap using the GRS and BUHMBOX approaches

\begin{tabular}{|c|c|c|c|c|c|c|}
\hline Cohort data & Test trait & $\begin{array}{l}\text { Number } \\
\text { of SNPs }\end{array}$ & $\begin{array}{c}\text { GRS } \\
P \text { value }\end{array}$ & $\begin{array}{c}\text { GRS } \beta \\
(95 \% \mathrm{Cl})\end{array}$ & $\begin{array}{c}\text { BUHMBOX } \\
P \text { value }\end{array}$ & $\begin{array}{c}\text { BUHMBOX } \\
\text { power at } \pi=0.20\end{array}$ \\
\hline \multirow[t]{11}{*}{ T1D } & $A A$ & 10 & $1.4 \times 10^{-120}$ & $0.76(0.69-0.82)$ & 0.83 & 0.15 \\
\hline & ATD & 7 & $1.4 \times 10^{-31}$ & $0.48(0.40-0.56)$ & 0.30 & 0.05 \\
\hline & CEL & 38 & $2.2 \times 10^{-35}$ & $0.32(0.27-0.38)$ & 0.16 & 0.50 \\
\hline & CRO & 119 & $2.4 \times 10^{-5}$ & $0.08(0.04-0.11)$ & 0.54 & 0.99 \\
\hline & JIA & 22 & $3.6 \times 10^{-151}$ & $0.44(0.40-0.47)$ & 0.37 & 0.96 \\
\hline & PBC & 19 & $1.1 \times 10^{-12}$ & $0.16(0.11-0.20)$ & 0.18 & 0.82 \\
\hline & PSC & 12 & $4.1 \times 10^{-26}$ & $0.38(0.31-0.45)$ & 0.91 & 0.08 \\
\hline & RA & 68 & $6.6 \times 10^{-89}$ & $0.55(0.49-0.60)$ & 0.45 & 0.40 \\
\hline & SJO & 7 & $3.9 \times 10^{-146}$ & $0.53(0.49-0.57)$ & 0.84 & 0.66 \\
\hline & SLE & 16 & $1.1 \times 10^{-83}$ & $0.44(0.39-0.48)$ & 0.79 & 0.91 \\
\hline & VIT & 12 & $2.5 \times 10^{-90}$ & $0.59(0.53-0.65)$ & 0.14 & 0.33 \\
\hline \multirow[t]{11}{*}{ RA } & $A A$ & 10 & $1.5 \times 10^{-22}$ & $0.28(0.22-0.34)$ & 0.71 & 0.23 \\
\hline & AS & 24 & $6.1 \times 10^{-4}$ & $0.10(0.04-0.15)$ & 0.19 & 0.20 \\
\hline & ATD & 7 & $3.9 \times 10^{-20}$ & $0.34(0.27-0.41)$ & 0.57 & 0.08 \\
\hline & CEL & 38 & $6.4 \times 10^{-20}$ & $0.21(0.17-0.26)$ & 0.57 & 0.63 \\
\hline & $J I A$ & 22 & $8.9 \times 10^{-125}$ & $0.36(0.33-0.39)$ & 0.61 & 0.99 \\
\hline & PBC & 19 & $1.5 \times 10^{-13}$ & $0.15(0.11-0.19)$ & 0.83 & 0.90 \\
\hline & PSC & 12 & $6.2 \times 10^{-14}$ & $0.24(0.18-0.31)$ & 0.46 & 0.12 \\
\hline & SLE & 16 & $4.3 \times 10^{-06}$ & $0.10(0.05-0.14)$ & 0.34 & 0.96 \\
\hline & SSC & 5 & $9.6 \times 10^{-10}$ & $0.22(0.15-0.29)$ & 0.08 & 0.09 \\
\hline & T1D & 53 & $9.6 \times 10^{-207}$ & $0.43(0.40-0.46)$ & 0.29 & 1.00 \\
\hline & VIT & 12 & $1.8 \times 10^{-11}$ & $0.18(0.12-0.23)$ & 0.02 & 0.41 \\
\hline $\begin{array}{l}\text { Seronegative } \\
\text { RA }\end{array}$ & $\begin{array}{l}\text { Seropositive } \\
\text { RA }\end{array}$ & 14 & $1.1 \times 10^{-10}$ & $0.30(0.21-0.39)$ & 0.008 & 0.26 \\
\hline MDD & $\mathrm{SCZ}$ & 90 & $1.5 \times 10^{-5}$ & $0.17(0.09-0.24)$ & 0.28 & 0.53 \\
\hline
\end{tabular}

SCZ, schizophrenia. Only the traits that have significant GRS $P$ values in the positive direction are shown. Significant GRS $P$ value indicates evidence of shared genetic structure; significant BUHMBOX $P$ value indicates evidence of heterogeneity. See Supplementary Table 4 for the full results for all traits tested.

large numbers of loci with even nominal evidence of association in addition to established genome-wide significant loci (Supplementary Fig. 4 and Supplementary Note).

\section{Controlling for linkage disequilibrium}

Although BUHMBOX adequately controlled the FPR when loci were truly independent, we were concerned that long-range LD between apparently independent loci may introduce false positives ${ }^{29}$. To ensure that BUHMBOX was robust to $\mathrm{LD}$, we implemented the following strategies: (i) stringent $\mathrm{LD}$ pruning of $\mathrm{D}_{\mathrm{B}}$-associated loci to exclude SNPs with $r^{2}>0.1$ and (ii) accounting for any remaining residual LD by assessing the relative increase of correlations in cases as compared to controls (delta correlations). We evaluated these strategies by measuring FPR using the RA Immunochip Consortium data ${ }^{30}$. In 1,000 different loosely pruned $\left(r^{2}<0.5\right)$ SNP sets constructed using the Sweden EIRA data (Online Methods), the FPR without using delta correlations was high $(22.4 \%$ at $P<0.05)$. Applying delta correlations reduced this FPR to 9.5\%. When we used stringent pruning $\left(r^{2}<0.1\right)$, FPR was appropriately controlled (FPR was 5.9\% and 5.3\% with and without delta correlations, respectively). Although LD pruning alone was sufficiently effective for FPR control in this simulation, we have used both strategies throughout the paper to be conservative.

\section{Accounting for population stratification}

Another potential confounding factor is population stratification. If population stratification exists, weak correlations between unlinked loci may occur, leading to inappropriate significance. If similar population stratification exists in cases and controls, the use of delta correlations mitigates this effect. To more aggressively control for the effect of stratification at the individual level, we implemented BUHMBOX to regress out principal components from risk allele dosages before calculating correlation statistics. To evaluate this strategy, we simulated extreme population stratification using HapMap ${ }^{31}$ data $(60 \mathrm{CEU}$ and 60 YRI founders as cases and $90 \mathrm{JPT}$ plus $\mathrm{CHB}$ founders as controls; $\lambda_{\mathrm{GC}}=26.5$ ). It did not surprise us that, in 5,000 randomly sampled sets of independent SNPs, we observed an inflated BUHMBOX FPR $(14.1 \%$ at $P<0.05)$. After regressing out the effects of ten principal components from risk allele dosages, we observed that the FPR was appropriately controlled $(5.7 \%$ at $P<0.05)$. As an additional test under a more realistic scenario, we merged genotype data from northern Europe (Sweden EIRA cohort; 2,762 cases and 1,940 controls) and southern Europe (Spain cohort; 807 cases and 399 controls) in the RA Immunochip Consortium case-control data set $^{30}$ (Online Methods) to create a highly stratified data set. In 1,000 sets of randomly sampled independent SNPs, we observed an inflation of the FPR $(8.6 \%$ at $P<0.05)$; this was appropriately corrected (FPR of $5.9 \%$ at $P<0.05)$ when we regressed out the effects of ten principal components.

\section{Application to autoimmune diseases}

Autoimmune diseases have genetic risk loci in common $2,4,32-36$ that cluster in specific immune pathways $2,27,36$. We used the GRS approach to evaluate shared genetic structure for autoimmune diseases and then applied BUHMBOX to assess heterogeneity. We obtained individual-level genotype data from the Type 1 Diabetes Genetics Consortium (T1DGC) UK case-control cohort (6,670 cases and 9,416 controls) ${ }^{37}$ and the RA Immunochip Consortium's six RA case-control cohorts (7,279 seropositive RA cases and 15,870 controls) ${ }^{30}$ (Online Methods). We evaluated genetic sharing for a spectrum of autoimmune diseases with T1D and RA. We obtained independent associated loci for all 18 autoimmune diseases $\left(r^{2}<0.1\right.$, including MHC SNPs) from ImmunoBase (see URLs and Supplementary Table 3) and tested the association of GRSs for these autoimmune diseases with T1D and RA case status.

We observed substantial genetic sharing between autoimmune diseases. T1D demonstrated significant sharing with alopecia areata (AA), autoimmune thyroid disease (ATD), celiac disease (CEL), Crohn's disease (CRO), juvenile idiopathic arthritis (JIA), primary biliary cirrhosis (PBC), primary sclerosing cholangitis (PSC), RA, Sjögren's syndrome (SJO), systemic lupus erythematosus (SLE), and vitiligo (VIT) (positive association, $P<1 \times 10^{-4}$ ). RA exhibited significant genetic sharing with AA, ankylosing spondylitis (AS), ATD, CEL, JIA, PBC, PSC, SLE, systemic sclerosis (SSC), T1D, and VIT $\left(P<1 \times 10^{-3}\right)$. Overall, GRSs showed significant positive associations for 11 autoimmune diseases each in the T1D and RA cohorts (GRS $P<2.9 \times 10^{-3}=0.05 / 17$, correcting for 17 diseases tested; Table 1 and Supplementary Table 4). We considered only these traits for subsequent analyses. To evaluate the degree of heterogeneity necessary to achieve the observed genetic sharing for these autoimmune diseases, we calculated the GRS regression coefficient, which we previously showed approximates the expected $\pi$ (ref. 38), assuming no pleiotropy. 

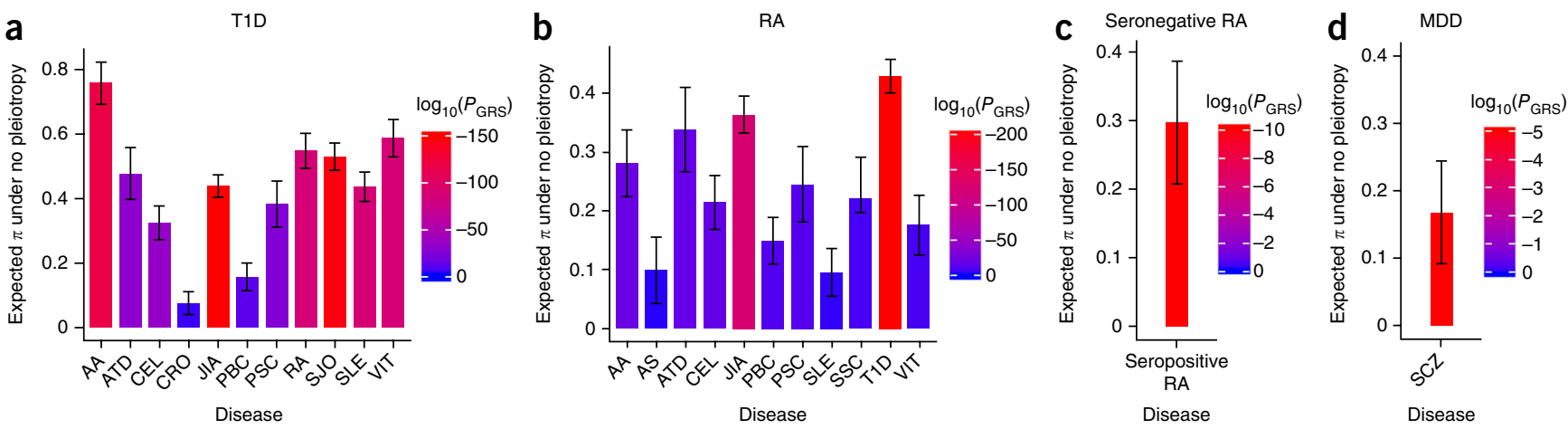

Figure 4 Genetic sharing among autoimmune diseases and psychiatric disorders. (a-d) In each plot, the $y$ axis shows the heterogeneity proportion $\pi$ value expected to explain the observed genetic sharing. Error bars denote $95 \%$ confidence intervals. Heterogeneity proportion estimates were based on GRS analysis, assuming no pleiotropy for T1D (a), RA (b), seronegative RA (c), and MDD (d). In a and b, we show only the diseases of the 17 tested that had significantly positive GRS $P$ values.

On the basis of the GRS coefficients, we observed $\pi$ estimates ranging from 0.08 to 0.76 across the different autoimmune diseases in T1D and from 0.10 to 0.43 in RA (Fig. 4 and Table 1).

We estimated the power of BUHMBOX to detect heterogeneity, correcting for 11 tests $\left(P<4.5 \times 10^{-3}\right)$. BUHMBOX was well powered for some autoimmune traits; at $\pi=0.2$, four traits had $>90 \%$ power for T1D and four traits had $>90 \%$ power for RA (Fig. 5). Despite this, we observed no evidence of heterogeneity at all (corrected $P>0.2$; Fig. 6 and Table 1). Our findings suggest that autoimmune diseases have risk alleles and pathways in common with T1D and RA, and this is not caused by subgroups of genetically similar cases resulting from misclassifications or molecular subtypes.

\section{Application to subtype misclassifications in RA}

RA consists of two subtypes, seropositive and seronegative RA, with distinct clinical outcomes and $\mathrm{MHC}$ associations ${ }^{38}$. Patients are classified into these two subtypes by whether they have serological evidence of antibodies reactive to cyclic citrullinated peptides (CCP). Although testing for antibody to CCP is specific, its lack of sensitivity can result in some patients with seropositive RA being misclassified as seronegative for $\mathrm{RA}^{20}$. We have previously demonstrated that there is shared genetic structure for seropositive and seronegative RA using the GRS approach $^{38}$, which could imply misclassifications of up to $26.3 \%$ of cases between the two RA subtypes.

We used BUHMBOX to evaluate whether seropositive RA misclassifications were present in a seronegative RA cohort. We used the seronegative RA cohort (2,406 cases and 15,870 controls) from the RA Immunochip Consortium ${ }^{30}$. Among 68 independent RA-associated loci, we chose SNPs that were associated with seropositive RA $\left(P<5 \times 10^{-8}\right)$ but not with seronegative RA $\left(P>5 \times 10^{-8}\right)$ in our Immunochip data. This criterion resulted in the selection of 14 specific loci exclusively associated with seropositive RA (Supplementary Table 3). The seropositive RA GRS was significantly associated with seronegative RA case status $\left(\beta=0.30, P=1.1 \times 10^{-10}\right)$. The regression coefficient $(\beta=0.30)$ represents an upper bound for $\pi$ (Fig. 4). BUHMBOX suggested that heterogeneity was indeed present $(P=0.008$; Fig. 6 , Table 1, and Supplementary Table 4), consistent with potential subtype misclassifications. As a more stringent test, we selected SNPs on the basis of the results from a test for heterogeneity between the RA subtypes; for this test, we obtained $P$ values by assigning individuals with seropositive RA as cases and individuals with seronegative RA as controls. We chose both SNPs that were associated with seropositive RA $\left(P<5 \times 10^{-8}\right)$ and were nominally significant in the heterogeneity test between the RA subtypes $(P<0.05$; Supplementary Table 3$)$. Applying BUHMBOX to these 12 loci still showed significant heterogeneity in the seronegative RA cohort $(P=0.017)$.

\section{Application to major depressive disorder and schizophrenia}

Current definitions of psychiatric disorders reflect clinical syndromes, with overlapping clinical features. As a result, psychiatric diagnoses for a patient may change as their symptoms evolve ${ }^{21}$. In addition to the potential for misdiagnosis, a subset of true MDD cases may be genetically more similar to schizophrenia. If heterogeneity with respect to schizophrenia risk alleles exists among MDD cases, then genetic studies would suggest evidence of coheritability for the two disorders ${ }^{17}$, as has been observed in previous studies ${ }^{3,6,7}$. The unintentional inclusion a

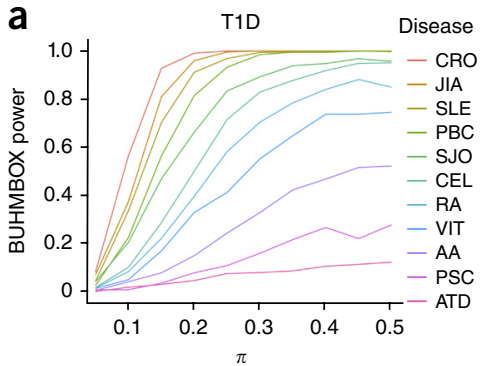

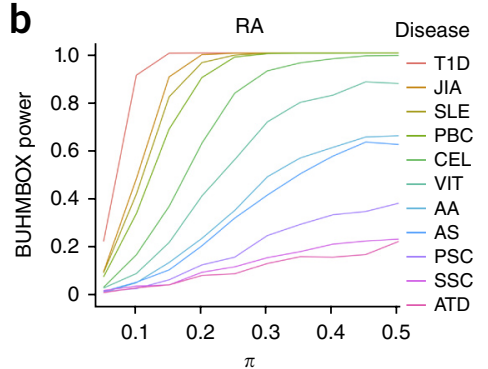
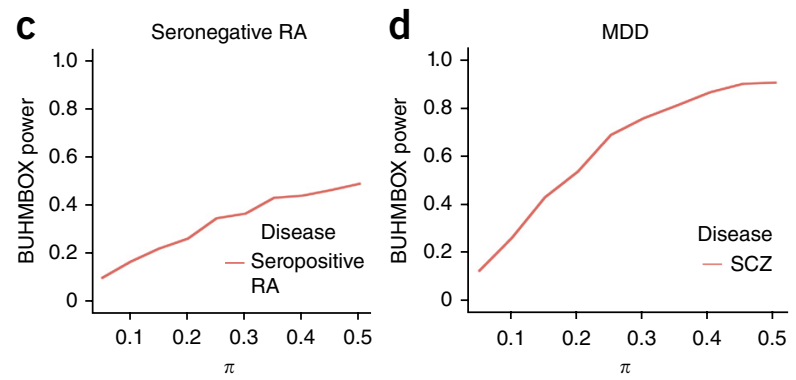

Figure 5 Statistical power of BUHMBOX to detect heterogeneity. (a-d) Power calculated by performing 1,000 simulations with the corresponding sample size, number of risk alleles, RAFs, and ORs for T1D (a), RA (b), seronegative RA (c), and MDD (d). To calculate power for $\mathbf{c}$ and d, we used a significance threshold of 0.05 . For $\mathbf{a}$ and $\mathbf{b}$, the threshold was adjusted using Bonferroni correction accounting for 11 tests each in T1D and RA. 
Figure 6 BUHMBOX results. (a-d) Results for diseases with significantly positive GRS $P$ values in T1D (a), RA (b), seronegative RA (c), and MDD (d) (for complete results for all traits tested, see Supplementary Table 4). Significant GRS $P$ values indicate evidence of shared genetic structure; significant BUHMBOX $P$ values indicate evidence of heterogeneity. Point size represents the number of $D_{B}$-associated SNPs included in the analysis. Dashed vertical lines denote the Bonferroni-adjusted significance threshold for the BUHMBOX test statistic. The arrow indicates a significant BUHMBOX test statistic.

of 'schizophrenia-like' MDD cases, owing to diagnostic misclassification or genetically distinct subgroups, has been acknowledged and explored as a potential source of bias in coheritability studies by previous investigators ${ }^{3,17}$.

We used BUHMBOX to test for a subgroup of schizophrenia-like cases in MDD. If a subset of MDD cases were misdiagnosed and in fact had schizophrenia or were more genetically similar to schizophrenia, we would expect to see subgroup heterogeneity among MDD cases with respect to schizophrenia risk loci. We first evaluated evidence of shared genetic structure among 90 known schizophrenia-associated loci ${ }^{39}$ (Supplementary Table 3) in 9,238 MDD cases and 7,521 controls from the Major Depressive Disorder Working Group of the Psychiatric Genomics Consortium $^{40}$ (Supplementary Table 5). Consistent with previous findings (Supplementary Table 6) ) $^{3,6,7}$, the schizophrenia GRS was associated with MDD case status $\left(P=1.54 \times 10^{-5}\right)$, indicating shared genetic structure (Fig. 4). For the GRS analysis, we used a refined subset of the total sample (6,382 MDD cases and 5,614 controls), excluding samples that overlapped with the schizophrenia GWAS ${ }^{39}$ (Online Methods). Application of cross-trait $\operatorname{LDSC}^{7}$ to estimate the genetic correlation provided further evidence of shared genetic structure between MDD and schizophrenia $\left(r_{\mathrm{g}}=0.47\right.$, standard error (s.e.) $\left.=0.07, P=1.61 \times 10^{-10}\right)$, of similar magnitude to previous reports ${ }^{7}$. However, the BUHMBOX $P$ value was not significant $(P=0.28)$, indicating no excess positive correlations among schizophrenia-associated loci in MDD cases (Fig. 6 and Supplementary Table 4). Our findings suggest no evidence of a subgroup of schizophrenia-like MDD cases. However, we note that we lacked adequate statistical power to detect heterogeneity in the context of a small heterogeneity proportion. Given the MDD sample size and the number of currently known schizophrenia risk loci, there was $53 \%$ power at $\pi=0.20$ but only $25 \%$ power at $\pi=0.10$ (Fig. 5).

\section{DISCUSSION}

BUHMBOX can be used to determine whether shared genetic structure between traits is the consequence of heterogeneity or pleiotropy on the basis of SNP genotype data alone. It can help to interpret recent observations of shared genetic structures in complex traits, including autoimmune, neuropsychiatric, and metabolic diseases. The intuition behind BUHMBOX is that, if heterogeneity exists, independent loci will show nonrandom positive correlations. Hence, correcting for population structure and long-range LD is critical for this approach to be effective. We emphasize that it is necessary to appropriately interpret the source of heterogeneity, which will depend on the biological b
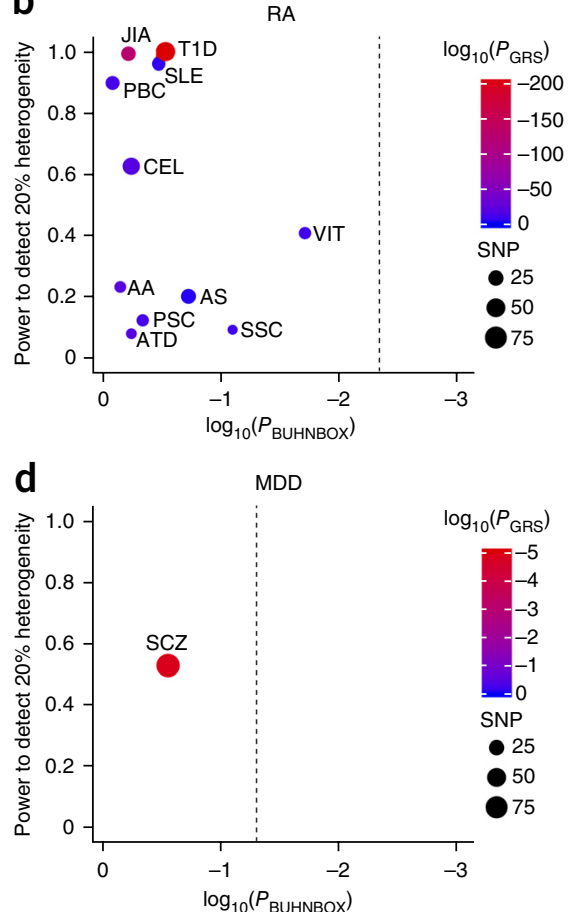

and clinical relationship between the two traits. We provide detailed information to guide interpretation in the Supplementary Note.

We demonstrated that genetic sharing among autoimmune diseases is due to pleiotropy, noting that for a few traits we had only modest power (Fig. 5). One notable exception was seronegative RA, which might contain misclassified seropositive RA cases. The results presented here demonstrate that seronegative RA is a heterogeneous phenotype with respect to genetic overlap with seropositive RA, bringing clarity to an ongoing debate about the nature of this disease. In contrast, we were underpowered to draw more definitive conclusions as to whether a subset of MDD cases are genetically similar to schizophrenia cases; as MDD cohorts increase in size, we will be able to reassess more accurately whether smaller proportions of heterogeneity might partially explain observed coheritability. Our results are consistent with recent analyses concluding that pleiotropy among psychiatric diseases is unlikely to be explained by misclassifications alone ${ }^{17}$.

We showed that the power of BUHMBOX is a function of sample size, $\pi$, and the number, effect sizes, and allele frequencies of risk loci. Power to detect subtle heterogeneity $(\pi<0.1)$ in current data sets is limited. However, in future studies, increasing sample size and the number of known associated loci will augment power. One potential strategy to augment power is to use a polygenic modeling ${ }^{3,12,13}$ approach, including a larger number of SNPs with less stringent significance thresholds (Supplementary Fig. 4 and Supplementary Note).

BUHMBOX has certain key caveats. First, it is designed to detect a specific type of heterogeneity resulting from the presence of a subgroup comprising a known second trait. Thus, BUHMBOX cannot currently be applied agnostically to detect the presence of heterogeneity in a data set. Second, BUHMBOX requires prior knowledge of associated loci and their effect sizes. For diseases with few known associated loci, BUHMBOX may perform suboptimally. Also, if known effect size estimates are inaccurate, power may decrease because appropriate weighting is crucial (Fig. 2). Third, BUHMBOX requires individual-level genotype data for a limited number of loci. Fourth, BUHMBOX can be sensitive to confounding factors. We recommend careful control of LD 
and population structure using LD pruning and principal components. Fifth, interpretation of the BUHMBOX test statistic is not simplistic. Positive findings indicate the presence of heterogeneity, but the results cannot distinguish between the various causes of this (for example, misclassifications, molecular subtypes, mediated pleiotropy, and ascertainment bias), and negative findings may indicate no heterogeneity or low power. To aid interpretation, BUHMBOX provides a power calculation based on sample size and risk allele information, but it may not always be accurate. For example, if pleiotropy and heterogeneity coexist, power may be overestimated. Sixth, if $\pi$ is small (for example, $0.05)$, BUHMBOX's ability to detect heterogeneity is limited. We expect that $\pi$ will vary between situations, and further clinical and biological investigations are necessary to uncover the true $\pi$. Finally, there is the unlikely possibility that real epistasis can manifest as positive signal in BUHMBOX. Broadly, BUHMBOX can be thought of as capturing a specific form of epistasis where risk alleles correlate positively within the additive model. As such, if this specific form of epistasis occurs naturally between $D_{B}$-associated SNPs and if this epistasis structure is shared with $\mathrm{D}_{\mathrm{A}}$, it has the potential to create a significant BUHMBOX test result and confound these analyses. However, this specific type of epistasis seems unlikely; were it present, application of BUHMBOX using $\mathrm{D}_{\mathrm{B}}$-associated SNPs in $\mathrm{D}_{\mathrm{B}}$ cases to detect apparent 'heterogeneity' might yield a significant result.

When comparing BUHMBOX to existing approaches, we focused on the GRS method. However, the results of our comparison also apply to other existing methods such as mixed-model-based approaches ${ }^{5,6}$ and LD-score-based approaches ${ }^{7}$, which are similar to the GRS approach in the sense that they detect both pleiotropy and heterogeneity. We expect that BUHMBOX will complement any of these methods to facilitate interpretation of observed genetic sharing between traits. Our statistical approach may be extended to have application beyond heterogeneity, including identification of missing heritability resulting from this type of heterogeneity ${ }^{41}$. These applications will become more feasible as functional annotations of SNPs advance in the coming years.

URLs. BUHMBOX software, https://www.broadinstitute.org/mpg/ buhmbox/; ImmunoBase, http://www.immunobase.org/.

\section{METHODS}

Methods and any associated references are available in the online version of the paper.

Note: Any Supplementary Information and Source Data files are available in the online version of the paper.

\section{ACKNOWLEDGMENTS}

This work was supported in part by funding from the US National Institutes of Health (NIH) (1R01AR063759 (S.R.), 1R01AR062886 (S.R.), 1UH2AR067677-01 (S.R.), and U19AI111224-01 (S.R.)) and Doris Duke Charitable Foundation grant 2013097. B.H. is supported by the Asan Institute for Life Sciences, Asan Medical Center, Seoul, Republic of Korea (2016-0717) and the Korean Health Technology R\&D Project, Ministry of Health and Welfare, Republic of Korea (HI14C1731). J.G.P. is supported by Fulbright Canada, the Weston Foundation, and Brain Canada through the Canada Brain Research Fund. K.S. is supported by an NIH training grant (T32HG002295). N.R.W. is supported by the Australian National Health and Medical Research Council (1087889 and 1078901). This research uses resources provided by the Type 1 Diabetes Genetics Consortium, a collaborative clinical study sponsored by the National Institute of Diabetes and Digestive and Kidney Diseases (NIDDK), National Institute of Allergy and Infectious Diseases (NIAID), National Human Genome Research Institute (NHGRI), National Institute of Child Health and Human Development (NICHD), and Juvenile Diabetes Research Foundation International (JDRF) and supported by grant U01DK062418.

\section{AUTHOR CONTRIBUTIONS}

B.H. and S.R. conceived the statistical approach and organized the project. B.H., J.G.P., and S.R. led and coordinated analyses and wrote the initial manuscript. E.S. and N.R.W. provided guidance on the statistical approach. K.S., C.H.L., D.D., X.H., Y.R.P., and E.K. contributed to the implementation of specific analyses and offered feedback on the statistical methodologies. P.K.G., S.R.D., J.W., J.M., S.E., L.K., S.R., and T.H. contributed RA samples and insight on the clinical implications to RA. W.-M.C., S.O.-G., and S.S.R. contributed T1D samples and insight on clinical implications to T1D. The Major Depressive Disorder Working Group contributed MDD samples and insight on the clinical implications to MDD. All authors contributed to the final manuscript.

\section{COMPETING FINANCIAL INTERESTS}

The authors declare no competing financial interests.

Reprints and permissions information is available online at http://www.nature.com/ reprints/index.html.

1. Sivakumaran, S. et al. Abundant pleiotropy in human complex diseases and traits. Am. J. Hum. Genet. 89, 607-618 (2011).

2. Cotsapas, C. et al. Pervasive sharing of genetic effects in autoimmune disease. PLoS Genet. 7, e1002254 (2011).

3. Cross-Disorder Group of the Psychiatric Genomics Consortium. Identification of risk loci with shared effects on five major psychiatric disorders: a genome-wide analysis. Lancet 381, 1371-1379 (2013).

4. Fortune, M.D. et al. Statistical colocalization of genetic risk variants for related autoimmune diseases in the context of common controls. Nat. Genet. 47, 839-846 (2015).

5. Lee, S.H., Yang, J., Goddard, M.E., Visscher, P.M. \& Wray, N.R. Estimation of pleiotropy between complex diseases using single-nucleotide polymorphism-derived genomic relationships and restricted maximum likelihood. Bioinformatics 28 , 2540-2542 (2012).

6. Cross-Disorder Group of the Psychiatric Genomics Consortium. Genetic relationship between five psychiatric disorders estimated from genome-wide SNPs. Nat. Genet. 45, 984-994 (2013).

7. Bulik-Sullivan, B. et al. An atlas of genetic correlations across human diseases and traits. Nat. Genet. 47, 1236-1241 (2015).

8. Pendergrass, S.A. et al. Phenome-wide association study (PheWAS) for detection of pleiotropy within the Population Architecture using Genomics and Epidemiology (PAGE) Network. PLoS Genet. 9, e1003087 (2013)

9. Collins, F.S. \& Varmus, H. A new initiative on precision medicine. N. Engl. J. Med 372, 793-795 (2015).

10. Criswell, L.A. et al. Analysis of families in the Multiple Autoimmune Disease Genetics Consortium (MADGC) collection: the PTPN22 620W allele associates with multiple autoimmune phenotypes. Am. J. Hum. Genet. 76, 561-571 (2005).

11. Kendler, K.S., Neale, M.C., Kessler, R.C., Heath, A.C. \& Eaves, L.J. Major depression and generalized anxiety disorder. Same genes, (partly) different environments? Arch. Gen. Psychiatry 49, 716-722 (1992).

12. Wray, N.R., Goddard, M.E. \& Visscher, P.M. Prediction of individual genetic risk to disease from genome-wide association studies. Genome Res. 17, 1520-1528 (2007).

13. International Schizophrenia Consortium. Common polygenic variation contributes to risk of schizophrenia and bipolar disorder. Nature 460, 748-752 (2009).

14. Lee, S.H. et al. New data and an old puzzle: the negative association between schizophrenia and rheumatoid arthritis. Int. J. Epidemiol. 44, 1706-1721 (2015).

15. Power, R.A. et al. Polygenic risk scores for schizophrenia and bipolar disorder predict creativity. Nat. Neurosci. 18, 953-955 (2015).

16. Solovieff, N., Cotsapas, C., Lee, P.H., Purcell, S.M. \& Smoller, J.W. Pleiotropy in complex traits: challenges and strategies. Nat. Rev. Genet. 14, 483-495 (2013).

17. Wray, N.R., Lee, S.H. \& Kendler, K.S. Impact of diagnostic misclassification on estimation of genetic correlations using genome-wide genotypes. Eur. J. Hum. Genet. 20, 668-674 (2012).

18. Silverberg, M.S. et al. Diagnostic misclassification reduces the ability to detect linkage in inflammatory bowel disease genetic studies. Gut 49, 773-776 (2001).

19. van der Linden, M.P. et al. Value of anti-modified citrullinated vimentin and thirdgeneration anti-cyclic citrullinated peptide compared with second-generation anti-cyclic citrullinated peptide and rheumatoid factor in predicting disease outcome in undifferentiated arthritis and rheumatoid arthritis. Arthritis Rheum. 60, 2232-2241 (2009).

20. Wiik, A.S., van Venrooij, W.J. \& Pruijn, G.J. All you wanted to know about anti-CCP but were afraid to ask. Autoimmun. Rev. 10, 90-93 (2010).

21. Bromet, E.J. et al. Diagnostic shifts during the decade following first admission for psychosis. Am. J. Psychiatry 168, 1186-1194 (2011).

22. Gibson, P. et al. Subtypes of medulloblastoma have distinct developmental origins. Nature 468, 1095-1099 (2010)

23. Smoller, J.W., Lunetta, K.L. \& Robins, J. Implications of comorbidity and ascertainment bias for identifying disease genes. Am. J. Med. Genet. 96, 817-822 (2000).

24. Burrell, R.A., McGranahan, N., Bartek, J. \& Swanton, C. The causes and consequences of genetic heterogeneity in cancer evolution. Nature 501, 338-345 (2013). 
25. Jeste, S.S. \& Geschwind, D.H. Disentangling the heterogeneity of autism spectrum disorder through genetic findings. Nat. Rev. Neurol. 10, 74-81 (2014).

26. Flint, J. \& Kendler, K.S. The genetics of major depression. Neuron 81, 484-503 (2014).

27. Cho, J.H. \& Feldman, M. Heterogeneity of autoimmune diseases: pathophysiologic insights from genetics and implications for new therapies. Nat. Med. 21, 730-738 (2015).

28. Welter, D. et al. The NHGRI GWAS Catalog, a curated resource of SNP-trait associations. Nucleic Acids Res. 42, D1001-D1006 (2014).

29. Raychaudhuri, S. et al. Genetic variants at CD28, PRDM1 and CD2/CD58 are associated with rheumatoid arthritis risk. Nat. Genet. 41, 1313-1318 (2009).

30. Eyre, S. et al. High-density genetic mapping identifies new susceptibility loci for rheumatoid arthritis. Nat. Genet. 44, 1336-1340 (2012).

31. International HapMap Consortium. The International HapMap Project. Nature 426, 789-796 (2003).

32. Smyth, D.J. et al. Shared and distinct genetic variants in type 1 diabetes and celiac disease. N. Engl. J. Med. 359, 2767-2777 (2008).

33. Festen, E.A. et al. A meta-analysis of genome-wide association scans identifies IL18RAP, PTPN2, TAGAP, and PUS10 as shared risk loci for Crohn's disease and celiac disease. PLoS Genet. 7, e1001283 (2011).
34. Zhernakova, A. et al. Meta-analysis of genome-wide association studies in celiac disease and rheumatoid arthritis identifies fourteen non-HLA shared loci. PLoS Genet. 7, e1002004 (2011).

35. Jostins, L. et al. Host-microbe interactions have shaped the genetic architecture of inflammatory bowel disease. Nature 491, 119-124 (2012).

36. Cotsapas, C. \& Hafler, D.A. Immune-mediated disease genetics: the shared basis of pathogenesis. Trends Immunol. 34, 22-26 (2013).

37. Onengut-Gumuscu, S. et al. Fine mapping of type 1 diabetes susceptibility loci and evidence for colocalization of causal variants with lymphoid gene enhancers. Nat. Genet. 47, 381-386 (2015).

38. Han, B. et al. Fine mapping seronegative and seropositive rheumatoid arthritis to shared and distinct HLA alleles by adjusting for the effects of heterogeneity. Am. J. Hum. Genet. 94, 522-532 (2014).

39. Schizophrenia Working Group of the Psychiatric Genomics Consortium. Biological insights from 108 schizophrenia-associated genetic loci. Nature 511, 421-427 (2014).

40. Ripke, S. et al. A mega-analysis of genome-wide association studies for major depressive disorder. Mol. Psychiatry 18, 497-511 (2013).

41. Wray, N.R. \& Maier, R. Genetic basis of complex genetic disease: the contribution of disease heterogeneity to missing heritability. Curr. Epidemiol. Rep. 1, 220-227 (2014). 


\section{ONLINE METHODS}

Genetic risk score approach. Given $M$ independent risk loci associated with $\mathrm{D}_{\mathrm{B}}$, we calculated the GRS of individual $i$ as

$$
\mathrm{GRS}_{i}=\sum_{j=1}^{M} x_{i j} \beta_{j}
$$

where $x_{i j}$ is the risk allele dosage for individual $i$ at marker $j$ and $\beta_{j}$ is the effect size (log-transformed OR) of the risk allele at marker $j$ for disease $\mathrm{D}_{\mathrm{B}}$. The GRS approach calculates GRSs for all individuals and associates GRSs with case/control status of $\mathrm{D}_{\mathrm{A}}$. In the logistic regression framework for associating GRSs and $D_{A}$ status, we can obtain the regression coefficient for the GRS ( $\left.\beta_{\mathrm{GRS}}\right)$. We previously showed that $\beta_{\mathrm{GRS}}$ approximates the proportion of $\mathrm{D}_{\mathrm{A}}$ cases that are genetically $D_{B}$ cases (heterogeneity proportion, $\pi$ ) if we assume that there is no pleiotropy and the GRS association is solely driven by a subgroup ${ }^{38}$. Thus, $\beta_{\mathrm{GRS}}$ represents an upper bound of $\pi$.

The BUHMBOX approach. To detect heterogeneity within $\mathrm{D}_{\mathrm{A}}$ cases driven by a subgroup that is genetically similar to $D_{B}$ cases, we used the following procedure. (i) We prepared genotype data for $\mathrm{D}_{\mathrm{A}}$ cases and controls and information about SNPs associated with $\mathrm{D}_{\mathrm{B}}$ (risk allele, RAF, and OR). (ii) We pruned SNPs associated with $\mathrm{D}_{\mathrm{B}}$ on the basis of LD in control samples (excluding SNPs with $r^{2}>0.1$ or $\pm 1 \mathrm{Mb}$ from other SNPs). (iii) We obtained the risk allele dosages of the pruned SNPs in $\mathrm{D}_{\mathrm{A}}$ cases and controls. (iv) We regressed out principal components from risk allele dosages to obtain residual dosages, one locus at a time. (v) We calculated $\mathbf{R}$, the correlation matrix of the residual dosages of risk alleles in $N$ cases with $\mathrm{D}_{\mathrm{A}}$ and $\mathbf{R}^{\prime}$, in $N^{\prime}$ controls. (vi) We calculated $\mathbf{Y}$, a matrix whose nondiagonal elements were $z$ scores from delta correlations

$$
\mathbf{Y}=\sqrt{\frac{N \times N^{\prime}}{N+N^{\prime}}}\left(\mathbf{R}-\mathbf{R}^{\prime}\right)
$$

(vii) We calculated the BUHMBOX statistic

$$
S_{\text {BUHMBox }}=\frac{\sum_{i<j} w_{i j} y_{i j}}{\sqrt{\sum_{i<j} w_{i j}^{2}}}
$$

where $y_{i j}$ is the element in $\mathbf{Y}$ in row $i$ and column $j$. Given $M$ pruned SNPs, $(i, j)$ iterates $M(M-1) / 2$ nondiagonal elements of $\mathbf{Y}$. The $w_{i j}$ term is a weighting function that is designed to maximize power, such that (equation (13) in the Supplementary Note)

$$
w_{i j}=\frac{\sqrt{p_{i}\left(1-p_{i}\right) p_{j}\left(1-p_{j}\right)}\left(\gamma_{i}-1\right)\left(\gamma_{j}-1\right)}{\left(\left(\gamma_{i}-1\right) p_{i}+1\right)\left(\left(\gamma_{j}-1\right) p_{j}+1\right)}
$$

where $p_{i}$ is the RAF of SNP $i$ and $\gamma_{i}$ is the OR of SNP $i$ for $\mathrm{D}_{\mathrm{B}}$. The BUHMBOX statistic follows a $N(0,1)$ distribution under the null hypothesis. We calculated the significance of this statistic as a positive one-sided test; the $P$ value is $P_{\text {BUhmBoX }}=1-\Phi\left(S_{\text {BUhmBoX }}\right)$, where $\Phi$ is the cumulative density function of the standard normal distribution. In the context of heterogeneity, excessive positive correlations among $\mathrm{D}_{\mathrm{B}}$ risk alleles in $\mathrm{D}_{\mathrm{A}}$ cases result in $P_{\mathrm{BUHMBOX}}<\alpha$. See Supplementary Table 1 for a comparison of the BUHMBOX and GRS approaches. The BUHMBOX test statistic was inspired by previous work deriving covariance between correlation estimates ${ }^{42}$ and on combining dependent estimates ${ }^{43,44}$. For details of the intuition, derivation, optimization, and interpretation of the BUHMBOX test statistic, see the Supplementary Note.

Code availability. BUHMBOX has been fully implemented as a publicly available R script (see URLs).

Power and false positive rate simulations. Given the sample size of $D_{A}$ cases $(N)$, the proportion of $D_{A}$ cases that actually show genetic characteristics of $\mathrm{D}_{\mathrm{B}}$ cases (heterogeneity proportion, $\pi$ ), and the number of risk loci associated with $\mathrm{D}_{\mathrm{B}}(M)$, we simulated studies to estimate the power of our method as follows. To simulate a reasonable joint distribution of RAFs and ORs, we downloaded the GWAS catalog (as of 29 April 2014). Among all binary traits in the catalog, we selected traits with $\geq 50$ reported associated SNPs, resulting in 22 traits with 1,480 SNPs. From these SNPs, we sampled $M$ pairs of RAFs $(p)$ and their corresponding ORs $(\gamma)$. To simulate genotypes, we set the RAF to $\gamma p /((\gamma-1) p+1)$ for a subgroup ( $N \pi$ individuals) and to $p$ for the other subgroup $(N(1-\pi)$ individuals) because $N \pi$ individuals could be thought of as $\mathrm{D}_{\mathrm{B}}$ cases. Within each subgroup, we generated genotypes assuming that risk alleles were distributed according to Hardy-Weinberg equilibrium and that risk loci were independent. We assumed Hardy-Weinberg equilibrium in cases because we assumed an additive disease model. Then, we applied BUHMBOX to calculate the $P$ value. We repeated this 1,000 times to approximate power as the proportion of simulations with $P \leq 0.05$. We evaluated power for different values of $N, M$, and $\pi$.

Under the assumption that the risk loci were independent, the FPR simulation was equivalent to the power simulation described above, with the only difference being that $\pi$ was set to 0 , which forced the null hypothesis. We measured the FPR by assuming $N=1,000$ and $M=20$ and constructing $1,000,000$ such studies.

Linkage disequilibrium simulations. To simulate realistic LD, we used chromosome 22 data from control individuals in the Swedish EIRA cohort of the RA data set $(2,762 \text { cases and 1,940 controls })^{30}$. We assigned half of control individuals as cases and the rest as controls. To generate 1,000 random sets of SNPs, we began from all SNPs and thinned the SNP set by tenfold with different seed numbers using PLINK ${ }^{45}$ (with the command option --thin 0.1). We then pruned each of the 1,000 data sets using PLINK ${ }^{45}$ with an $r^{2}$ criterion of 0.5 or 0.1 .

Population stratification simulations. To assess the effects of population stratification, we conducted two sets of simulations. For the first, we used data from HapMap ${ }^{31}$ release 23 (60 CEU founders, 60 YRI founders, and 90 JPT + CHB founders), setting CEU + YRI individuals as cases and JPT + $\mathrm{CHB}$ individuals as controls. We calculated principal components after LD pruning $\left(r^{2}<0.1\right)$. For $\mathrm{D}_{\mathrm{B}}$-associated SNPs, we randomly selected 5,000 sets of 22 independent SNPs; we selected a single SNP from each autosome. For the second simulation, we used genotype data from a northern European RA cohort (Swedish EIRA; 2,762 cases and 1,940 controls) and a southern Europe cohort (Spain; 807 cases and 399 controls) from the RA data set $^{30}$. For this simulation, we used SNPs that we had generated for LD simulations (described above; thinned from Swedish EIRA chromosome 22 with criterion $r^{2}<0.1$ ), by setting the Swedish samples as cases and adding the Spanish samples as controls.

Application to specific phenotypes. Type 1 diabetes data set. To evaluate pleiotropy and heterogeneity among 18 autoimmune diseases and T1D, we applied the GRS and BUHMBOX approaches to the UK case-control data set provided by the T1DGC ${ }^{37}$, which consisted of 16,086 samples $(6,670$ cases and 9,416 controls) from three collections: (i) cases from the UK-GRID, (ii) shared controls from the British 1958 Birth Cohort, and (iii) shared controls from Blood Services controls (data release 4 February 2012; hg18). The samples were collected from 13 regions. All samples were collected after obtaining informed consent, and samples were genotyped on the Immunochip array. GRS and BUHMBOX analyses were conducted using the region index as a covariate.

Rheumatoid arthritis data set. To evaluate pleiotropy and heterogeneity between 18 autoimmune diseases and RA, we used the RA Immunochip Consortium data from six RA case-control cohorts (UK, US, Dutch, Spanish, Swedish UMEA, and Swedish EIRA) ${ }^{30}$. To evaluate pleiotropy in autoimmune diseases, we used 7,279 seropositive RA cases and 15,870 controls. To evaluate misclassifications of RA subtypes, we used 2,406 seronegative RA samples and the same controls. Seropositive and seronegative RA cases were defined in each cohort using standard clinical practices to assess whether patients were reactive to antibody against $\mathrm{CCP}^{38}$. All samples were obtained with informed consent and were collected through protocols approved by an institutional review board. All individuals self-reported as white and of European descent. Samples were genotyped with the Immunochip array. We merged the data from six cohorts into one and used binary variables representing cohorts as well as ten principal components as covariates in the analysis. 
Defining autoimmune risk loci. We accessed ImmunoBase (7 June 2015 version) to define genome-wide significant risk loci for 18 autoimmune diseases. We did not include inflammatory bowel disease, owing to its redundancy with Crohn's disease and ulcerative colitis. For each of the 18 autoimmune diseases analyzed, we pruned the list of index SNPs obtained from ImmunoBase in PLINK ${ }^{45}$ with options --r2 --ld-window-r2 0.1, using the 1000 Genomes Project Phase 1 European reference panel for LD. For all pairs of SNPs with $r^{2}>0.1$, we kept the most strongly associated SNP. To ensure completely independent risk loci, we also removed SNPs annotated as being located in the same chromosomal region in ImmunoBase, again keeping the most strongly associated index SNP (Supplementary Table 3). When a locus was not in the Immunochip data sets, we looked for a proxy $\left(r^{2}>0.2\right)$ on the basis of the 1000 Genomes Project data.

Major depressive disorder data set. We used BUHMBOX to investigate the relationship between MDD and schizophrenia, which have been previously reported to have common genetic etiology on the basis of polygenic risk scoring $^{3}$ and coheritability analyses ${ }^{6}$. The full MDD sample analyzed comprised nine GWAS data sets collected from eight separate studies (Supplementary Table 5) as previously described ${ }^{40}$. All samples were collected through protocols approved by an institutional review board and were obtained with informed consent. Independence of the training (schizophrenia) and target (MDD) data sets is crucial in GRS analyses; GRSs are constructed using effect size estimates obtained using allele frequency differences between cases and controls in the training GWAS, and overlapping cases or controls will therefore bias the association of GRSs to the target data set in the positive direction. In contrast, the BUHMBOX test statistic is based on the correlation of risk allele dosages among cases, which is orthogonal to allele frequency differences in cases and controls and is therefore not inflated by sample overlap. Thus, for the GRS analysis, individual MDD samples (4 cases and 886 controls) that overlapped with samples in the schizophrenia GWAS ${ }^{39}$ were removed from the analysis; three GWAS cohorts with an insufficient number of independent control samples $(N<5)$ were also removed from the analysis. GRS analyses were conducted in each of the remaining six GWAS data sets (Supplementary Table 5), followed by meta-analysis of the GRSs. To obtain the overall GRS effect size $(\beta)$ and test statistic, we used the inverse-variance-weighted fixedeffects method. For BUHMBOX, we used the full data set; analyses were conducted in each of the nine GWAS data sets (Supplementary Table 5) followed by meta-analysis. Because the BUHMBOX statistic is a $z$ score, we performed meta-analysis of the BUHMBOX results across the data sets using the standard weighted-sum $z$-score approach, where $z$ scores are weighted by the square root of the sample size.

Defining schizophrenia risk loci. Schizophrenia-associated SNPs were selected as those showing genome-wide significant association with schizophrenia $\left(P<5 \times 10^{-8}\right)$ in the most recent Psychiatric Genomics Consortium ${ }^{39}$ GWAS. For schizophrenia-associated SNPs not directly genotyped in the MDD GWAS data sets, we selected proxy SNPs as those with the highest $r^{2}$ value from the list of all proxies with $r^{2}>0.2$ using the 1000 Genomes Project Phase 1 European reference panel. Of the 97 schizophrenia-associated SNPs (11 indels were not considered in our analysis), $90 \mathrm{LD}$-independent SNPs $\left(r^{2}>0.1,>1\right.$ $\mathrm{Mb}$ away from each other) were available for analysis in the MDD GWAS data sets either via direct genotyping or by genotyping of a proxy SNP (see Supplementary Table 3 for a detailed list of SNPs).

42. Jennrich, R.I. An asymptotic $\chi^{2}$ test for the equality of two correlation matrices J. Am. Stat. Assoc. 65, 904-912 (1970).

43. Wei, L.J., Lin, D.Y. \& Weissfeld, L. Regression analysis of multivariate incomplete failure time data by modeling marginal distributions. J. Am. Stat. Assoc. 84 1065-1073 (1989).

44. Lin, D.Y. \& Sullivan, P.F. Meta-analysis of genome-wide association studies with overlapping subjects. Am. J. Hum. Genet. 85, 862-872 (2009).

45. Purcell, S. et al. PLINK: a tool set for whole-genome association and populationbased linkage analyses. Am. J. Hum. Genet. 81, 559-575 (2007). 